\title{
Forehead Regional Oxygen Saturation (rSO2)- Related Ear-Level Arterial Pressure and Lower Thigh rSO2 in the Steep Trendelenburg Position with CO2 Pneumoperitoneum and the Beach Chair Position
}

\author{
Tomoko Fukada $^{1}$, Yuri Tsuchiya ${ }^{1}$, Hiroko Iwakiri ${ }^{1}$, Makoto Ozaki ${ }^{1}$, Minoru Nomura ${ }^{1}$ \\ 1. Department of Anesthesiology, Tokyo Women's Medical University, School of Medicine, Tokyo, JPN
}

Corresponding author: Tomoko Fukada, blandsileo5719@gmail.com

\section{Abstract \\ Introduction}

Regional oxygen saturation (rSO2) reflects tissue perfusion. This observational study aimed to examine the change in the forehead and lower thigh rSO2 associated with intraoperative posture, anesthesia regimen, or mean arterial pressure (mAP) at heart and external auditory meatus (ear) levels.

\section{Methods}

Patients undergoing robot-assisted laparoscopic radical prostatectomy in the Trendelenburg position at $30^{\circ}$ with pneumoperitoneum (TPP) or arthroscopic shoulder surgery in the beach chair position at $70^{\circ}(\mathrm{BCP})$ under desflurane-remifentanil (D/R) or propofol-remifentanil $(\mathrm{P} / \mathrm{R})$ anesthesia were examined. Bilateral forehead and lower thigh $\mathrm{rSO} 2$ values and mean radial artery pressure were measured simultaneously at heart and ear levels.

\section{Results}

In TPP, there were no differences under anesthesia regimens in the forehead or lower thigh $\mathrm{rSO} 2$ change, although one patient with an absolute lower thigh $\mathrm{rSO} 2$ of $\leqslant 50 \%$ in the lithotomy position complained of transient limb pain. No correlation was observed between rSO2 and mAP. In BCP, forehead rSO2 decreased and lower thigh rSO2 increased under either of the anesthesia regimens. The coefficient of correlation between forehead $\mathrm{rSO} 2$ and heart-level and ear-level $\mathrm{mAP}$ was 0.341 and 0.236 , respectively.

\section{Conclusions}

Review began 05/30/2021 Review ended 06/06/2021 Published 06/16/2021

๑) Copyright 2021

Fukada et al. This is an open access article distributed under the terms of the Creative Commons Attribution License CC-BY 4.0., which permits unrestricted use, distribution, and reproduction in any medium, provided the original author and source are credited.
There were no differences under anesthesia regimens in the changes of forehead rSO2 and lower thigh rSO2. In TPP, significant changes in forehead rSO2 and lower thigh rSO2 were not observed. Monitoring lower thigh rSO2 might be useful for preventing lower extremity pain. In BCP, forehead rSO2 decreased and lower thigh rSO2 increased from the supine position to the BCP. To prevent brain damage, anesthesiologists should pay attention to heart- and ear-level mAP.

\section{Categories: Anesthesiology}

Keywords: regional oxygen saturation, forehead, lower thigh, mean arterial pressure, heart-level, ear-level

\section{Introduction}

Regional oxygen saturation (rSO2) reflects tissue perfusion and thus should be monitored closely during certain types of surgeries, especially cerebral rSO2. During cardiac surgery, a decrease in forehead rSO2 has been associated with cognitive dysfunction and delirium as well as prolonged hospital stay after surgery [13]. Similarly, frontal lobe $\mathrm{rSO} 2$ has been measured to predict cerebral ischemia during carotid endarterectomy and to detect postoperative stroke and cerebral hyperperfusion syndrome after carotid endarterectomy [4-6]. Certain surgical positions can exacerbate these changes in rSO2. For instance, robotassisted laparoscopic radical prostatectomy (RALP) is performed in the Trendelenburg position at $30^{\circ}$ with pneumoperitoneum (TPP), which affects the intracranial pressure (ICP) and thus alters cerebral blood flow (CBF) and bilateral forehead rSO2. Arthroscopic shoulder surgery in the beach chair position (BCP) may cause serious neurological injury owing to a decrease in cerebral perfusion $[7,8]$. Therefore, changes only in forehead rSO2 during RALP or BCP were measured [9-13]; only one study measured forehead rSO2 and lower thigh rSO2 during RALP [14].

Thus, our primary objective was to simultaneously examine the change in the forehead and lower thigh 
rSO2, associated with TPP or BCP. The secondary objective was to evaluate the influence of mean arterial pressure (mAP) at the heart level and the external auditory meatus (ear) level on forehead rSO2 change.

The change in rSO2 during RALP and arthroscopic shoulder surgery as shown in this study were presented as a poster at the International Society for Anaesthetic Pharmacology 26th Annual Meeting, on October 20, 2017.

\section{Materials And Methods}

\section{Patients}

Our study was approved by the institutional review board of Tokyo Women's Medical University (3945, 3945R1, and 3945-R2; approval date May 28, 2016; July 25, 2017; and March 22, 2019) and registered at University Hospital Medical Information Network Clinical Trials Registry (UMIN-CTR) on July 13, 2016

(UMIN000023155). Intravenous anesthetics were added in approval number 3945-R1, and the study period was extended in approval number $3945-\mathrm{R} 2$. Seventy-nine patients aged $\geqslant 20$ years undergoing RALP in TPP or arthroscopic shoulder surgery in BCP were enrolled from July 22, 2016, to November 25, 2019. The exclusion criteria were a history of cerebrovascular diseases or stenosis of the neck vessels. After we explained the study procedures and goals, we obtained written informed consent from all the patients.

\section{rSO2 measurement}

Four-channel rSO2 was measured using the INVOS ${ }^{\mathrm{TM}} 5100 \mathrm{C}$ Cerebral/Somatic Oximetry system and INVOS ${ }^{\text {TM }}$ 5100C Cerebral/Somatic Oximetry Adult Sensors (Medtronic, Minneapolis, Minnesota, USA). Before the induction of anesthesia, rSO2 sensors were affixed to the bilateral forehead and the lower thighs. The rSO2 levels of the bilateral forehead and lower thighs were measured during spontaneous breathing before anesthesia induction in the supine (baseline) position; after intubation; 0 (immediately), 5 , $10,20,30,60,90,120$, and 150 minutes after changing position; immediately and 10 minutes after returning to the supine position; and before discharge from the operating room. We evaluated the absolute rSO2 value at each measurement point and the number of cases in which rSO2 decreased to $\leqslant 80 \%$ of baseline or in which the absolute rSO2 decreased to $<50 \%$ in more than 5 minutes after a position change. The rSO2 values were only known to the data collector (AZ), who alerted the anesthesiologist when the absolute lower thigh rSO2 decreased to $\leqslant 50 \%$ during RALP.

\section{Anesthetic management}

Bispectral index (BIS; using the BIS A-2000 [Nihon Kohden, Tokyo, Japan]), noninvasive blood pressure of the arm, heart rate (HR), and peripheral oxygen saturation (SpO2) using the bedside monitor DS-8500 (Fukuda Denshi, Tokyo, Japan) were monitored simultaneously. Briefly, after induction of anesthesia, a 22G arterial catheter (BD Insyte-ATM, Nippon Becton Dickinson, Tokyo, Japan) was inserted percutaneously into the radial artery, and $\mathrm{mAP}$ was measured by positioned transducers at $5 \mathrm{~cm}$ below the sternal angle (heart level) and the external auditory meatus height (ear level).

Patients undergoing RALP were placed in the lithotomy position using Levitator@ (Mizuho, Tokyo, Japan) and in TPP after the insertion of medical instruments into their abdomen for prostatectomy. Intraabdominal pressure was maintained at $<15 \mathrm{mmHg}$. Patients undergoing arthroscopic shoulder surgery patients were placed in $\mathrm{BCP}$ at $70^{\circ}$ using the beach chair shoulder positioning system (Conmed, Utica, New York, USA), after tracheal intubation until the end of the operation. Arthroscopic shoulder surgery was performed under general anesthesia with interscalene brachial plexus block using levobupivacaine or ropivacaine, except in four cases, wherein patients did not accept regional anesthesia. The dose and timing of intravenous fentanyl administration during the operation and strategies for postoperative analgesia were at the discretion of the anesthesiologist.

Ten patients undergoing RALP and 10 undergoing arthroscopic shoulder surgery were anesthetized with desflurane-remifentanil (D/R) according to approval number 3945. The remaining patients undergoing each procedure were anesthetized with $\mathrm{D} / \mathrm{R}$ or propofol-remifentanil $(\mathrm{P} / \mathrm{R})$ anesthesia according to approval number 3945-R1. The administration of $\mathrm{D} / \mathrm{R}$ or $\mathrm{P} / \mathrm{R}$ was at the discretion of the anesthesiologists. None of the patients were premedicated. In the $\mathrm{D} / \mathrm{R}$ anesthesia group, general anesthesia was induced using intravenous $1-2 \mathrm{mg} / \mathrm{kg}$ propofol, followed by $0.1 \mathrm{mg}$ fentanyl, $0.5 \mu \mathrm{g} / \mathrm{kg} / \mathrm{min}$ remifentanil, and $0.6 \mathrm{mg} / \mathrm{kg}$ rocuronium for intubation. Anesthesia was maintained at a BIS of 40-60 with desflurane ( $<0.7$ minimum alveolar concentration) and $0.2-0.5 \mu \mathrm{g} / \mathrm{kg} / \mathrm{min}$ remifentanil. In the $\mathrm{P} / \mathrm{R}$ anesthesia group, anesthesia was induced with $4 \mu \mathrm{g} / \mathrm{mL}$ propofol using target-controlled infusion (TCI) and maintained at a BIS of 40-60 with TCI (Marsh model) using the Terfusion ${ }^{\circledR}$ TCI pump CoTE-371 (Terumo, Tokyo, Japan) and 0.2-0.5 $\mu \mathrm{g} / \mathrm{kg} / \mathrm{min}$ remifentanil. Rocuronium was administrated at $7 \mu \mathrm{g} / \mathrm{kg} / \mathrm{min}$ from intubation to the end of surgery in both anesthesia groups. The ventilator settings were as follows: tidal volume $8 \mathrm{~mL} / \mathrm{kg}$, inspiratory: expiratory ratio 1:2, inspired $\mathrm{O} 2$ fraction (FiO2) $45 \%$ with air, and positive end-expiratory pressure $5 \mathrm{cmH} 2 \mathrm{O}$. The respiratory rate was adjusted to maintain end-tidal $\mathrm{CO} 2$ (ETCO2) at 35-40 $\mathrm{mmHg}$. The rate of intravenous crystalloid infusion was kept constant at approximately $8 \mathrm{~mL} / \mathrm{kg} / \mathrm{h}$. After anesthesia induction, all patients wore surgical stockings and a foot pump (Kendall SCDTM 700, CardinalHealth, Ohio, USA) to prevent deep vein thrombosis. For patients whose pharyngeal or bladder temperature decreased to $<36.5^{\circ} \mathrm{C}$, a warmer 
blanket was used to prevent hypothermia. Patients who met discharge criteria with an Aldrete score of $\geqslant 9$ were moved to their respective wards. Postoperative cognitive dysfunction and delirium were checked through postoperative rounds and medical records.

During surgery, systolic arterial pressure (sAP) of $\leqslant 80 \mathrm{mmHg}$ at heart level was treated with ephedrine 4-8 $\mathrm{mg}$ or phenylephrine $0.05-0.1 \mathrm{mg}$, whereas sAP of $\geqslant 160 \mathrm{mmHg}$ at heart level was treated with nicardipine $0.5 \mathrm{mg}$. The administration of ephedrine or phenylephrine was at the discretion of anesthesiologists. The patients who were administered other cardiovascular agents were excluded because the interaction between these agents and anesthetics complicated the CBF and ICP changes [15].

\section{Statistical analysis}

The calculation of sample size was based on previous studies reporting that forehead rSO2 increased by a mean of $3 \%$ (standard deviation $[S D]=7 \%$ ) or decreased by a mean of $4 \%(S D=7 \%)$ after shifting from the supine position to RALP $[9,10,14]$ or BCP to maintained anesthesia using intravenous anesthetics or inhalation anesthetics $[8,12,16]$. For a power of 0.8 and a P-value of 0.05 , a sample size of 65 patients was calculated to enable the detection of a clinically relevant change in rSO2. All data are expressed as mean (SD). In each position, differences due to anesthesia regimen were compared using Pearson's chi-square test or Student's unpaired t-test. A repeated multivariate analysis of variance was performed to identify the association of anesthesia regimen with the time of change of rSO2. Changes from baseline were assessed by Dunnett's test. For all tests, $\mathrm{P}<0.05$ (two-tailed) was considered statistically significant. We calculated Pearson's correlation coefficient between rSO2 and mAP.

\section{Results}

Thirty-nine patients receiving RALP were assessed for eligibility; of these, two were excluded because of dopamine administration during surgery. Forty patients undergoing arthroscopic shoulder surgery patients were assessed for eligibility; among these, four were excluded because they were administered dopamine during surgery and three were not operated in BCP (Figure 1).
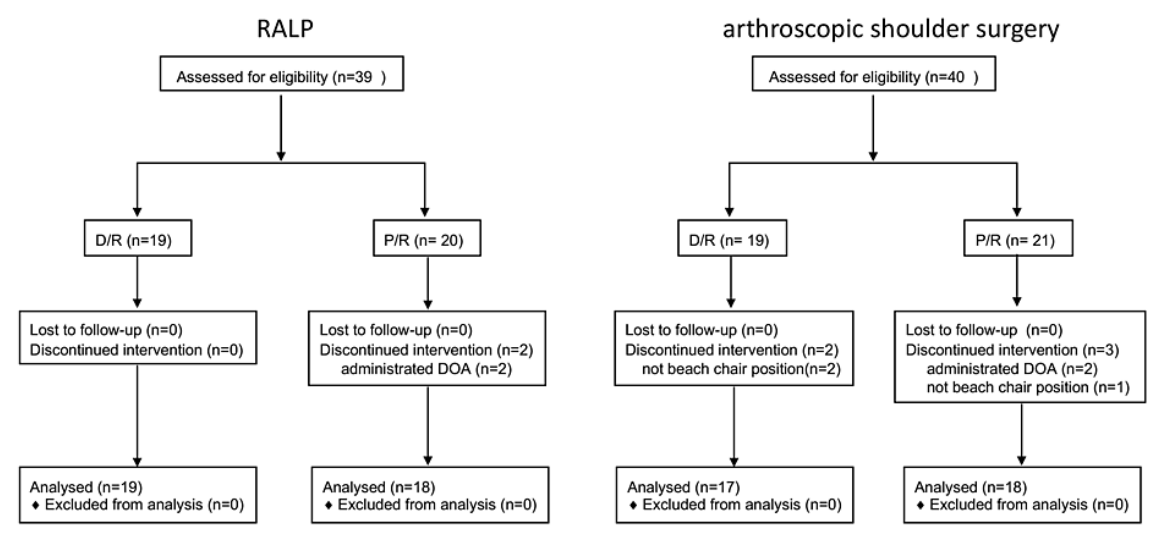

\section{FIGURE 1: Graphical depiction of subject flow through the study.}

RALP: robot-assisted laparoscopic radical prostatectomy, D/R: desflurane-remifentanil, P/R: propofolremifentanil, DOA: dopamine

According to approval number 3945, patients were anesthetized by D/R. After approval number 3945-R1, patients were anesthetized by $D / R$ or $P / R$, which was decided by the anesthesiologists.

Data were collected on 37 RALP patients in TPP (19 D/R anesthesia vs. $18 \mathrm{P} / \mathrm{R}$ anesthesia) and 35 arthroscopic shoulder surgery patients in BCP (17 D/R anesthesia vs. 18 P/R anesthesia). Forehead rSO2 was not compared with lower thigh rSO2 in either position because they were different organs. There were no bilateral differences in the forehead or lower thigh rSO2 under any condition, so we compared the average rSO2 values at the forehead and the lower thighs. Table 1 and Table 2 summarize the patients' characteristics, baseline data, and intraoperative clinical data. There were no significant differences in the baseline forehead and lower thigh rSO2 among all patients. 


\section{Cureus}

\begin{tabular}{|c|c|c|c|}
\hline & $D / R(n=19)$ & $P / R(n=18)$ & P-value \\
\hline Age (years) & $69(5)$ & $65(8)$ & 0.070 \\
\hline $\operatorname{Sex}(M / F)$ & $19 / 0$ & $18 / 0$ & - \\
\hline Height (cm) & $168(7)$ & $167(6)$ & 0.676 \\
\hline Weight (kg) & $69(8)$ & $69(9)$ & 0.925 \\
\hline ASA PS (I/II/III) & $3 / 14 / 2$ & $1 / 15 / 2$ & 0.604 \\
\hline Preoperative hemoglobin (g/dL) & $14.3(1.2)$ & $14.1(1.4)$ & 0.631 \\
\hline Comorbidities (HTN, DM, COPD) & 9 & 11 & 0.402 \\
\hline Baseline rSO2 (\%) forehead & $70(7)$ & $72(7)$ & 0.252 \\
\hline Baseline rSO2 (\%) lower thigh & $66(9)$ & $68(10)$ & 0.916 \\
\hline Baseline mAP (mmHg) & $110(14)$ & $115(16)$ & 0.282 \\
\hline Baseline HR (bpm) & $73(13)$ & 76 12) & 0.494 \\
\hline Duration of anesthesia (min) & $296(52)$ & 350 (103) & $0.048^{*}$ \\
\hline Duration of operation (min) & $220(53)$ & $261(109)$ & 0.150 \\
\hline Duration of TPP (min) & $205(54)$ & $243(83)$ & 0.129 \\
\hline Volume of infusion (mL) & 1936 (378) & $2121(552)$ & 0.267 \\
\hline Volume of bleeding $(\mathrm{mL})$ & $72(43)$ & $68(101)$ & 0.863 \\
\hline Volume of urine $(\mathrm{mL})$ & $238(217)$ & 317 (391) & 0.459 \\
\hline Total dose of remifentanil ( $\mu \mathrm{g} / \mathrm{kg} / \mathrm{min})$ & $0.31(0.09)$ & $0.32(0.06)$ & 0.567 \\
\hline Total dose of fentanyl $(\mu \mathrm{g} / \mathrm{kg} / \mathrm{min})$ & $0.03(0.01)$ & $0.03(0.01)$ & 0.859 \\
\hline Total dose of ephedrine $(\mu \mathrm{g} / \mathrm{kg} / \mathrm{min})$ & $0.28(0.3)$ & $0.47(0.40)$ & 0.895 \\
\hline Total dose of phenylephrine $(\mu \mathrm{g} / \mathrm{kg} / \mathrm{min})$ & $0.00(0.00)$ & $0.01(0.01)$ & 0.030 \\
\hline No of patients administrated ephedrine & 12 & 14 & 0.476 \\
\hline No of patients administrated phenylephrine & 3 & 10 & $0.049>>>3$ \\
\hline
\end{tabular}

TABLE 1: Patient characteristics, baseline data, and intraoperative clinical data in TPP.

Data are presented as mean (SD); ${ }^{*} \mathrm{P}<0.05$ by Student's unpaired test; ${ }^{* *} \mathrm{P}<0.05$ by Fisher's exact test

TPP: steep Trendelenburg position with pneumoperitoneum, D/R: desflurane-remifentanil, P/R: propofol-remifentanil, ASA PS: American Society of Anesthesia physical status, HTN: hypertension, DM: diabetes malleus, COPD: chronic obstructive pulmonary disease, TCI: target-controlled

infusion, rSO2: regional oxygen saturation, mAP: mean blood pressure, HR: heart rate 


\section{Cureus}

\begin{tabular}{|c|c|c|c|}
\hline & $D / R(n=17)$ & $P / R(n=18)$ & P-value \\
\hline Age (years) & $58(13)$ & 55 (14) & 0.538 \\
\hline $\operatorname{Sex}(M / F)$ & $9 / 8$ & $7 / 11$ & 0.404 \\
\hline Height (cm) & $162(11)$ & $163(12)$ & 0.684 \\
\hline Weight (kg) & $61(14)$ & 66 (14) & 0.247 \\
\hline ASA PS (I/II/III) & 7/9/1 & $6 / 12 / 0$ & 0.478 \\
\hline Preoperative hemoglobin (g/dL) & $14.0(1.5)$ & $13.7(1.4)$ & 0.537 \\
\hline Comorbidities (HTN, DM, COPD) & 2 & 6 & 0.637 \\
\hline Baseline rSO2 (\%) forehead & $66(11)$ & $70(7)$ & 0.833 \\
\hline Baseline rSO2 (\%) lower thigh & $67(8)$ & 73 (11) & 0.412 \\
\hline Baseline mAP (mmHg) & 105 (13) & $102(11)$ & 0.480 \\
\hline Baseline HR (bpm) & $68(11)$ & $69(11)$ & 0.749 \\
\hline Duration of anesthesia (min) & 207 (48) & $223(45)$ & 0.332 \\
\hline Duration of operation (min) & $120(40)$ & $119(49)$ & 0.935 \\
\hline Duration of BCP $(\mathrm{min})$ & $152(45)$ & $151(48)$ & 0.993 \\
\hline Volume of infusion (mL) & $1287(409)$ & $1519(416)$ & 0.106 \\
\hline Volume of bleeding (mL) & $6(7)$ & $5(5)$ & 0.678 \\
\hline Volume of urine $(\mathrm{mL})$ & $202(331)$ & $382(506)$ & 0.112 \\
\hline Total dose of remifentanil ( $\mu \mathrm{g} / \mathrm{kg} / \mathrm{min})$ & $0.27(0.20)$ & $0.22(0.09)$ & 0.332 \\
\hline Total dose of fentanyl $(\mu \mathrm{g} / \mathrm{kg} / \mathrm{min})$ & $0.03(0.02)$ & $0.03(0.01)$ & $0 / 388$ \\
\hline Total dose of ephedrine $(\mu \mathrm{g} / \mathrm{kg} / \mathrm{min})$ & $1.22(1.24)$ & $0.63(0.45)$ & 0.068 \\
\hline Total dose of phenylephrine $(\mu \mathrm{g} / \mathrm{kg} / \mathrm{min})$ & $0.01(0.02)$ & $0.03(0.04)$ & $0.028^{*}$ \\
\hline No of patients administrated ephedrine & 13 & 15 & 0.612 \\
\hline No of patients administrated phenylephrine & 7 & 12 & 0.40 \\
\hline
\end{tabular}

TABLE 2: Patient characteristics, baseline data, and intraoperative clinical data in BCP.

Data are presented as mean (SD); ${ }^{*} \mathrm{P}<0.05$ by Student's unpaired test; ${ }^{* *} \mathrm{P}<0.05$ by Fisher's exact test

BCP: beach chair position, D/R: desflurane-remifentanil, P/R: propofol-remifentanil, ASA PS: American society of anesthesia physical status, HTN: hypertension, DM: diabetes malleus, COPD: chronic obstructive pulmonary disease, TCl: target-controlled infusion, rSO2: regional oxygen saturation, mAP: mean arterial pressure, HR: heart rate

Figure 2 and Figure 3 present the changes in $\mathrm{mAP}$ at the heart and ear levels and heart rate (HR) during RALP in TPP, and during arthroscopic surgery in BCP. 


\section{Cureus}

A

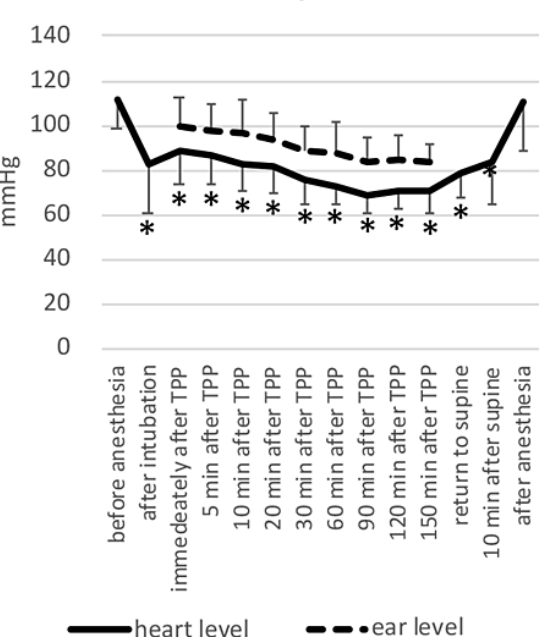

C

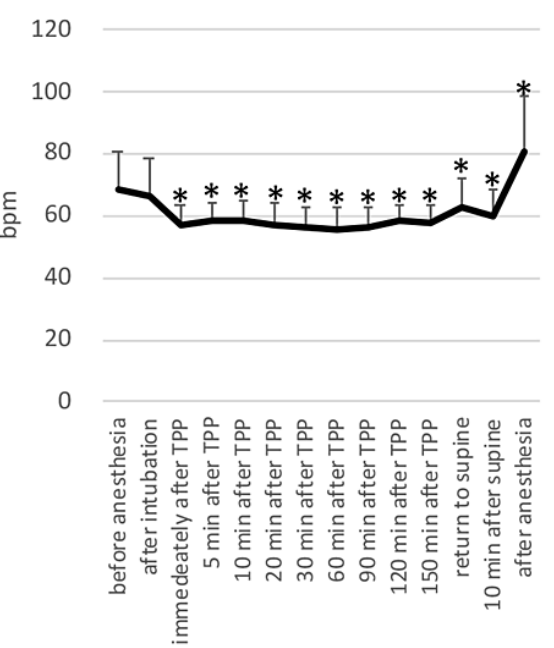

B $\quad \mathrm{mAP} P / \mathrm{R}$

140

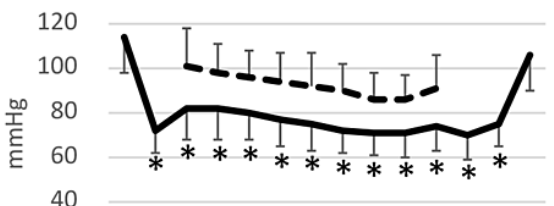

40

20

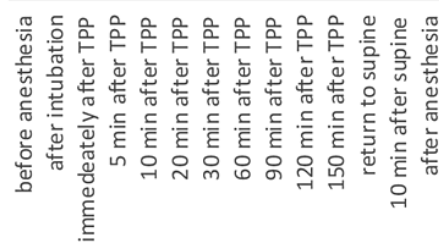

heart level - - - • ear level

D $\quad H R P / R$

120

100

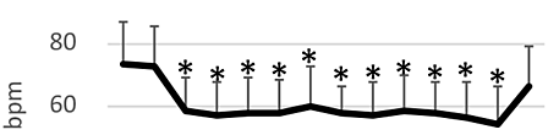

40

20

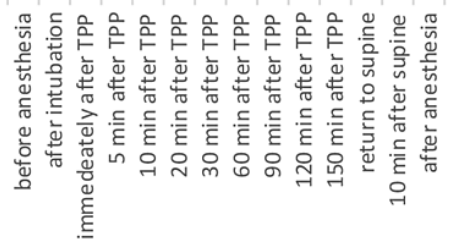

FIGURE 2: Changes in mean arterial pressure and heart rate during RALP.

Data represented by mean (error bar: SD); ${ }^{*}$ Compared with baseline values, $\mathrm{P}<0.05$

Figure 2A, 2B: Changes in mAP at the heart and ear levels under D/R or P/R, Figure 2C, 2D: Changes in HR under D/R or $\mathrm{P} / \mathrm{R}$

RALP: robot-assisted laparoscopic radical prostatectomy, mAP: mean arterial pressure, heart-level mAP: mAP measured at $5 \mathrm{~cm}$ under the sternal angle, ear-level mAP: mAP measured at external auditory meatus height, HR: heart rate, D/R: desflurane-remifentanil, P/R: propofol-remifentanil

There were no differences in MAP and HR between the anesthetic regimens. 


\section{Cureus}

A
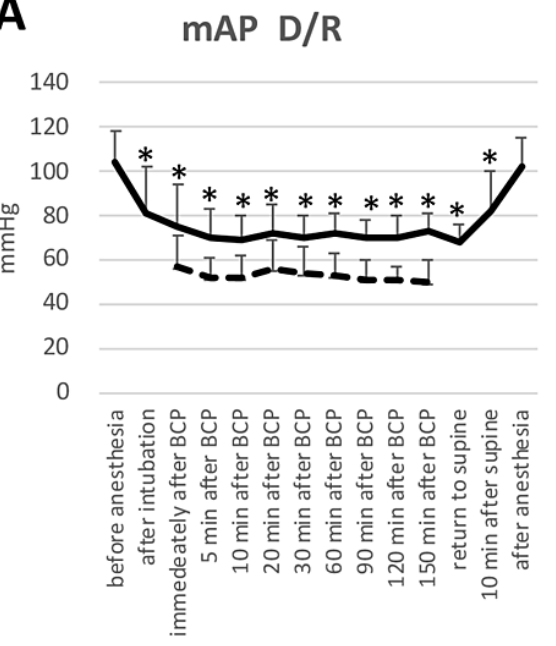

heart level

C

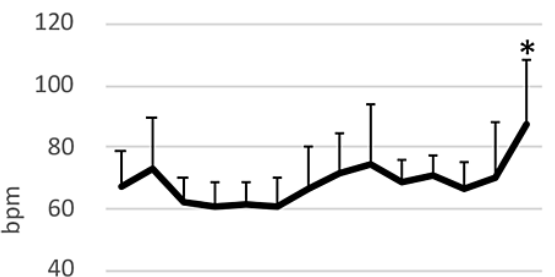

20

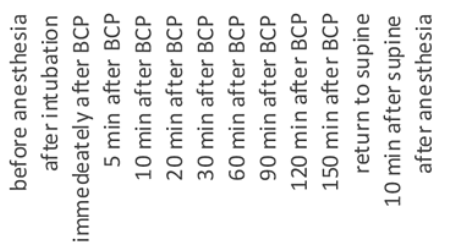

B

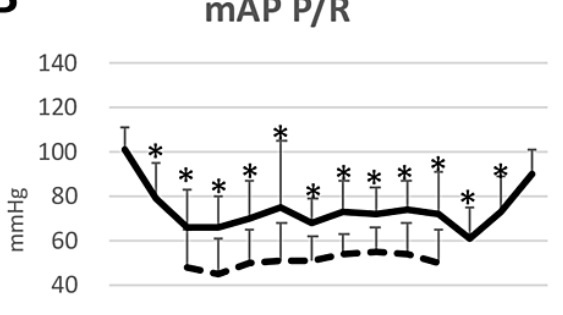

20

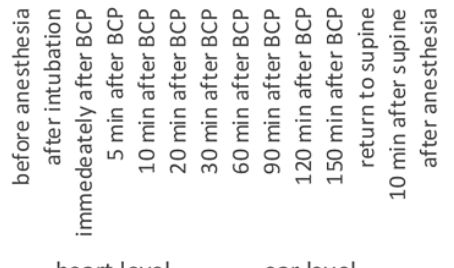

D HR P/R 120 100

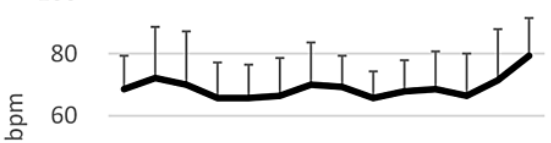

40

20

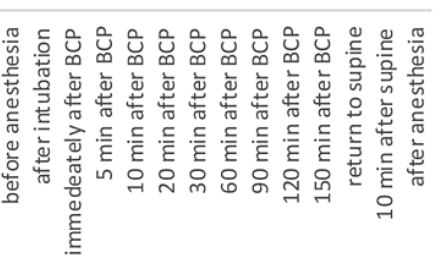

FIGURE 3: Changes in mean arterial pressure and heart rate during arthroscopic shoulder surgery.

Data represented by mean (error bar: SD), ${ }^{*}$ Compared with baseline values, $\mathrm{P}<0.05$

Figure 3A, 3B: Changes in mAP at the heart and ear levels under D/R or P/R, Figure 3C, 3D: Changes in HR under $\mathrm{D} / \mathrm{R}$ or $\mathrm{P} / \mathrm{R}$

mAP: mean arterial pressure, heart-level mAP: mAP measured at $5 \mathrm{~cm}$ under the sternal angle, ear-level mAP: mAP measured at external auditory meatus height, HR: heart rate, D/R: desflurane-remifentanil, P/R: propofol-remifentanil

There were no differences in MAP and HR between anesthetic regimens.

Ear-level transducers were 19 (5) cm lower than heart-level transducers in TPP and 22 (5) $\mathrm{cm}$ higher in BCP. As compared with heart-level mAP, ear-level mAP was 16 (4) mmHg higher in TPP and 19 (6) $\mathrm{mmHg}$ lower in BCP. Significant differences in mAP and HR were observed between elapsed time but not anesthesia regimens during each procedure. $\mathrm{SpO} 2$ was $\geqslant 95 \%$ in all cases.

The pharyngeal temperature of most patients was maintained at $35.8-36.5^{\circ} \mathrm{C}$ in TPP, although it was $<35.0$ ${ }^{\circ} \mathrm{C}$ in three patients. In BCP, the bladder temperature of all patients was maintained at $36.2-36.8{ }^{\circ} \mathrm{C}$. 


\section{Cureus}

\section{Change in rSO2 during RALP in TPP}

Changes in forehead rSO2 was associated with elapsed time but not with anesthesia regimens $(\mathrm{P}<0.0001$ and $\mathrm{P}=0.443$, respectively) (Figure $4 A, 4 B$ ). 


\section{Cureus}

A

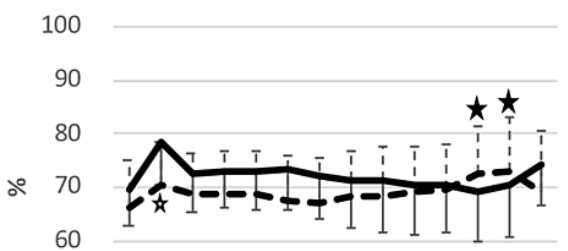

50

40

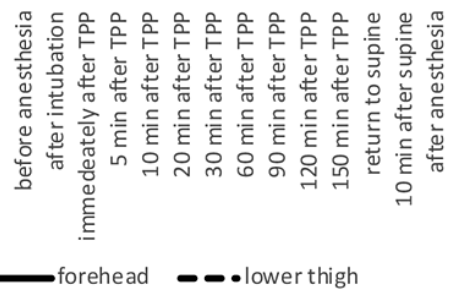

C $\mathrm{rSO}_{2} \mathrm{D} / \mathrm{R}$

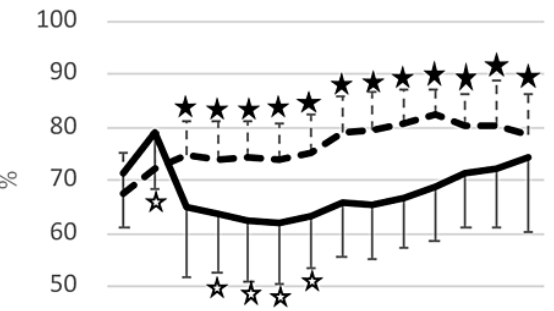

40

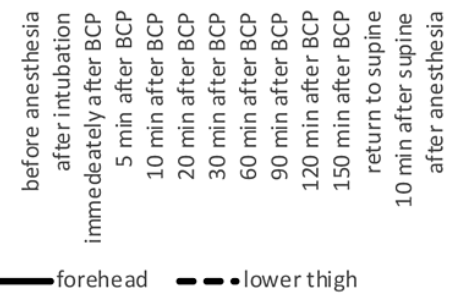

B $\quad \mathrm{rSO}_{2} \mathrm{P} / \mathrm{R}$

100

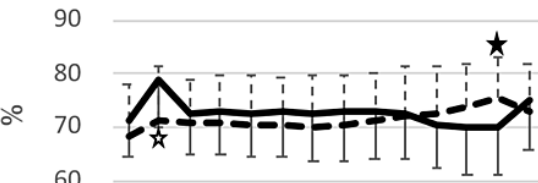

50

40

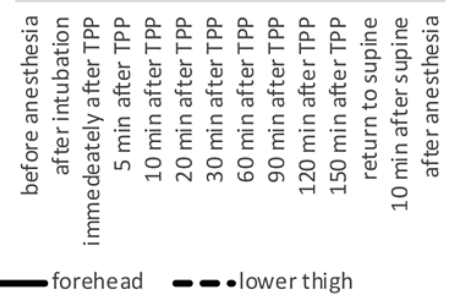

D $\quad \mathrm{rSO}_{2} \mathrm{P} / \mathrm{R}$

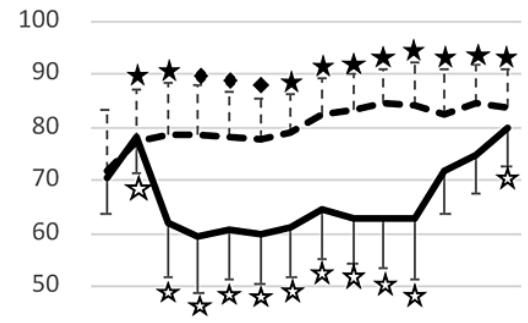

40

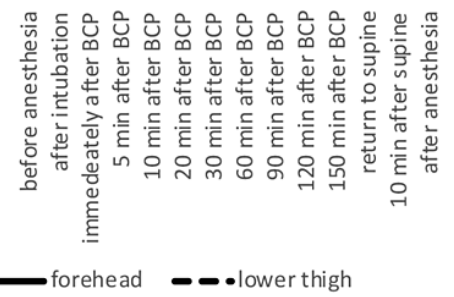

FIGURE 4: Changes in regional oxygen saturation during RALP and arthroscopic shoulder surgery.

Data represented by mean (error bar: SD)

Figures 4A, 4B: Changes in rSO2 under D/R or P/R in TPP, Figures 4C, 4D: Changes in rSO2 under D/R or P/R in $\mathrm{BCP}$

rSO2: regional oxygen saturation, RALP: robot-assisted laparoscopic radical prostatectomy, TPP: steep Trendelenburg position with pneumoperitoneum, BCP: beach chair position

Forehead rSO2 at each time as compared with baseline rSO2, $\mathrm{P}<0.05$, Lower thigh $\mathrm{rSO} 2$ as compared with baseline $\mathrm{rSO} 2$ at each time point, $\mathrm{P}<0.05$

During RALP, the mean (SD) baseline values of the forehead and lower thigh rSO2 under D/R anesthesia were $69 \%(7 \%)$ and $66 \%(9 \%)$, respectively. The baseline values of the forehead and lower thigh rSO2 under P/R anesthesia were $71 \%(7 \%)$ and $69 \%(10 \%)$, respectively.

During arthroscopic shoulder surgery, the baseline values of the forehead and lower thigh rSO2 under D/R anesthesia were $71 \%(10 \%)$ and $67 \%(8 \%)$, respectively. The baseline values of the forehead and lower thigh rSO2 under P/R anesthesia were $70 \%(7 \%)$ and $73 \%(11 \%)$, respectively.

Likewise, changes in lower thigh rSO2 were associated with elapsed time and not with anesthesia regimens 
$(\mathrm{P}<0.0001$ and $\mathrm{P}=0.568$, respectively). One patient exhibited a decrease in lower thigh $\mathrm{rSO} 2$ for one leg at $\leqslant 50 \%$ absolute value immediately after changing from the supine to lithotomy position and complained of limb pain immediately after anesthesia termination. In this case, the duration of TPP was 3 hours 21 minutes, and the intramuscular pressures of the lower limbs were not measured. His lower limb pain was relieved on the first postoperative day without fasciotomy, and potassium concentration was 3.8 $\mathrm{mEq} / \mathrm{L}$; creatine kinase was not measured. Although it is difficult to diagnose his limb pain as lower extremity compartment syndrome, on the basis of this experience, we adjusted the lower thigh/limb position after changing to TPP when the absolute lower thigh rSO2 decreased to $\leqslant 50 \%$. In fact, in four patients, after we rechecked the dorsalis pedis pulse, foot temperature, and knee and hip joint flexion, we changed the lower thigh/limb position in the Levitator@ to recover in lower thigh rSO2 to $>50 \%$. No correlation was noted between rSO2 and mAP (Table 3).

\begin{tabular}{|c|c|c|c|c|}
\hline & $\begin{array}{l}\text { Forehead } \mathrm{rSO}_{2} \text { and heart- } \\
\text { level mAP }\end{array}$ & $\begin{array}{l}\text { Forehead } \mathrm{rSO}_{2} \text { and ear- } \\
\text { level mAP }\end{array}$ & $\begin{array}{l}\text { Lower thigh } \mathrm{rSO}_{2} \text { and heart- } \\
\text { level mAP }\end{array}$ & $\begin{array}{l}\text { Lower thigh } \mathrm{rSO}_{2} \text { and ear- } \\
\text { level mAP }\end{array}$ \\
\hline TPP & 0.053 & 0.041 & -0.015 & 0.102 \\
\hline BCP & 0.341 & 0.236 & -0.093 & 0.005 \\
\hline
\end{tabular}

\section{TABLE 3: Correlation coefficients between rSO2 and mAP.}

TPP: steep Trendelenburg position with pneumoperitoneum, BCP: beach chair position, rSO2: regional oxygen saturation, mAP: mean blood pressure

There were no cases in which the forehead rSO2 decreased to $\leqslant 80 \%$ of baseline or in which the absolute rSO2 decreased to $\leqslant 50 \%$ at more than 5 minutes after a position change.

No patient exhibited postoperative cognitive dysfunction, delirium, or nerve damage of the lower limb after RALP, including the one patient who complained of lower limb pain immediately after anesthesia.

\section{Change in rSO2 during arthroscopic shoulder surgery in BCP}

Forehead rSO2 decreased and lower thigh rSO2 increased from the supine position to BCP under both anesthesia regimens. Changes in forehead $\mathrm{rSO} 2$ was dependent on elapsed time but not on anesthesia regimens ( $\mathrm{P}<0.0001$ and $\mathrm{P}=0.794$, respectively) (Figure $4 C, 4 D$ ). Likewise, changes in lower thigh $\mathrm{rSO} 2$ were associated with elapsed time and not with anesthesia regimens $(\mathrm{P}<0.0001$ and $\mathrm{P}=0.359$, respectively). We noted a weak correlation between forehead $\mathrm{rSO} 2$ and heart $\mathrm{mAP}$ and ear $\mathrm{mAP}$ : the correlation coefficient between forehead rSO2 and heart-level mAP and between forehead rSO2 and ear-level mAP were 0.341 and 0.236 , respectively (Table 3). In six patients receiving $\mathrm{D} / \mathrm{R}$ anesthesia and in eight patients receiving $\mathrm{P} / \mathrm{R}$ anesthesia, forehead $\mathrm{rSO} 2$ decreased to $\leqslant 80 \%$ of baseline at more than $5 \mathrm{~min}$ after a position change. The absolute rSO2 decreased to $<50 \%$ within $>5$ minutes after a position change in two patients in the $\mathrm{D} / \mathrm{R}$ anesthesia group and in five patients in the $\mathrm{P} / \mathrm{R}$ anesthesia group. None of the patients receiving arthroscopic shoulder surgery showed postoperative neurological complications.

\section{Discussion}

In clinical practice, bilateral forehead rSO2 is measured to detect brain hypoxemia or hyperperfusion [1-6]. Oxygen supply and demand determine rSO2. Hemoglobin concentration, hemoglobin saturation, local blood flow, and cardiac output (CO) are the predominant factors determining local oxygen supply, whereas body temperature, the depth of sedation, and pain are major factors determining oxygen demand. The mean preoperative hemoglobin was $14 \mathrm{~g} / \mathrm{dL}$, which underwent almost no change during surgery because of little blood loss. Hemoglobin saturation and the body temperature were maintained at $\geqslant 95 \%$ and $\sim 36-37^{\circ} \mathrm{C}$, respectively. Furthermore, the depth of sedation as measured by BIS of 40-60, and intraoperative pain were controlled by anesthetic delivery, and analgesics, respectively. Therefore, local blood flow and $\mathrm{CO}$ were important factors influencing the presence or absence of rSO2 change in TPP or BCP.

Generally, in normotensive individuals constant $\mathrm{CBF}$ is maintained by autoregulation within an mAP range of 50 (70)-150 mmHg, and the curves of autoregulation of CBF are flat between 50 (70)-150 $\mathrm{mmHg}$ [17]. By contrast, this curve shifts to the right on the BP axis in patients with uncontrolled hypertension, and this curve on the BP axis varies between the normal and right shifts in patients with well-controlled hypertension [18]. During TPP, no cases were observed in which forehead rSO2 decreased to $\leqslant 80 \%$ of the baseline or in which absolute rSO2 decreased to $\leqslant 50 \%$ for $>5$ minutes after a position change. Thus, mAP was within the flat section of the curves of autoregulation of CBF. In other words, CBF can also be determined by cerebral perfusion pressure (CPP), according to the previous reports, in which 170 minutes or 3 hours of TPP maintained CPP [19]. Similarly, compared with the supine position after induction of anesthesia, CO was maintained in TPP $[11,20]$. These studies supported our result of changes in forehead rSO2 during 150 
minutes in TPP.

No significant changes in lower thigh rSO2 at baseline were observed in TPP except in one case in which rSO2 decreased immediately after the patient position was changed from supine to lithotomy position accompanied by lower limb pain immediately after anesthesia. Because we handled this case, we adjusted the lower thigh/limb position in TPP when the absolute lower thigh rSO2 decreased to $\leqslant 50 \%$. Only one report, which measured cerebral and lower limb rSO2 simultaneously during RALP, showed that lower limb rSO2 increased [14]. Therefore, changing the lower thigh/limb position should be considered if the lower thigh rSO2 decreases.

During $\mathrm{BCP}$, the forehead rSO2 decreased and the lower thigh rSO2 increased from the supine position to the BCP under both anesthesia regimens. To prevent cerebral ischemia, maintaining CBF and CO is critical, particularly to maintain ear-level $\mathrm{mAP}$ at $>50 \mathrm{mmHg}$ or $>70 \mathrm{mmHg}[16,21]$. In this study, ephedrine or phenylephrine was administered to maintain heart-level sAP at $\geqslant 80 \mathrm{mmHg}$. This protocol may help to maintain ear-level $\mathrm{mAP}$ at $>50 \mathrm{mmHg}$, although it is impossible to maintain it at $>70 \mathrm{mmHg}$ in some cases. Therefore, the coefficient of correlation between forehead rSO2 and heart-level or ear-level mAP was 0.341 and 0.236 , respectively. Thus, to prevent cerebral ischemia, anesthesiologists should maintain ear-level $\mathrm{mAP}$ at $\geqslant 70 \mathrm{mmHg}$ even if monitoring only heart-level mAP is being monitored. Conversely, lower thigh rSO2 increased to about $80 \%$ by either of the anesthesia regimens, after changing to BCP because of vessel dilatation from the anesthetics.

We noted no difference in the change in forehead $\mathrm{rSO} 2$ by anesthesia regimen in the TPP and BCP. These results were supported by the previous studies in which the end-tidal concentration of desflurane was $<4 \%$ or the TCI level of propofol of $<2.5 \mu \mathrm{g} / \mathrm{mL}$ did not impair cerebral autoregulation and did not decrease CBF or CO [22-24].

There are some limitations to this study. First, we aimed to investigate the change in rSO2 in TPP and BCP, and we compared rSO2 for 150 minutes because the mean duration of BCP was 150 minutes. If we had investigated these for $>150$ minutes, we could have obtained different results. We observed the change in forehead rSO2 and lower thigh rSO2 at the same time; however, we did not evaluate these changes against each other because they are different organs. Moreover, we did not assess the preoperative or postoperative cognitive function using neuropsychologic tests. However, all patients met discharge criteria with an Aldrete score of $\geqslant 9$, and there were no obvious cognitive impairments during postoperative rounds or noted in the medical records. The major limitation of this study is that we did not measure CO even though we believed that mAP and CO were important factors that influence rSO2.

\section{Conclusions}

There were no differences under anesthesia regimens in the changes of forehead $\mathrm{rSO} 2$ and lower thigh $\mathrm{rSO} 2$. In TPP, significant changes in forehead rSO2 and lower-thigh rSO2 were not observed. Monitoring lower thigh rSO2 might be useful for preventing lower extremity pain. In BCP, forehead rSO2 decreased and lower thigh rSO2 increased from the supine position to the BCP. To prevent brain damage, anesthesiologists should pay attention to heart- and ear-level mAP.

\section{Additional Information \\ Disclosures}

Human subjects: Consent was obtained or waived by all participants in this study. Tokyo Women's Medical University issued approval 3945,3945-R1,3945-R2. Chancellor of Tokyo Women's Medical University approved our study; Does position of general anesthesia influence regional saturation of oxygen (rSO2)? Approval number 3945 Approval number 3945-R1 (addition of anesthesia method:total intravenous anesthesia by propofol) Approval number 3945-R2 (extended period of study). Animal subjects: All authors have confirmed that this study did not involve animal subjects or tissue. Conflicts of interest: In compliance with the ICMJE uniform disclosure form, all authors declare the following: Payment/services info: All authors have declared that no financial support was received from any organization for the submitted work. Financial relationships: All authors have declared that they have no financial relationships at present or within the previous three years with any organizations that might have an interest in the submitted work. Other relationships: All authors have declared that there are no other relationships or activities that could appear to have influenced the submitted work.

\section{Acknowledgements}

The authors thank Akiko Zaitsu, Department of Anesthesiology, Tokyo Women's Medical University, for allowing us to use the INVOSTM 5100C Cerebral/Somatic Oximetry system, for preparing the sensors and for collecting data.

\section{References}

1. de Tournay-Jetté E, Dupuis G, Bherer L, Deschamps A, Cartier R, Denault A: The relationship between 
cerebral oxygen saturation changes and postoperative cognitive dysfunction in elderly patients after coronary artery bypass graft surgery. J Cardiothorac Vasc Anesth. 2011, 25:95-104.

10.1053/j.jvca.2010.03.019

2. Colak Z, Borojevic M, Bogovic A, Ivancan V, Biocina B, Majeric-Kogler V: Influence of intraoperative cerebral oximetry monitoring on neurocognitive function after coronary artery bypass surgery: a randomized, prospective study. Eur J Cardiothorac Surg. 2015, 47:447-54. 10.1093/ejcts/ezu193

3. Slater JP, Guarino T, Stack J, et al.: Cerebral oxygen desaturation predicts cognitive decline and longer hospital stay after cardiac surgery. Ann Thorac Surg. 2009, 87:36-44; discussion 44-5. 10.1016/j.athoracsur.2008.08.070

4. Jonsson M, Lindström D, Wanhainen A, Djavani Gidlund K, Gillgren P: Near infrared spectroscopy as a predictor for shunt requirement during carotid endarterectomy. Eur J Vasc Endovasc Surg. 2017, 53:783-91. 10.1016/j.ejvs.2017.02.033

5. Cho JW, Jang JS: Near-infrared spectroscopy versus transcranial Doppler-based monitoring in carotid endarterectomy. Korean J Thorac Cardiovasc Surg. 2017, 50:448-52. 10.5090/kjtcs.2017.50.6.448

6. Pennekamp CW, Immink RV, den Ruijter HM, et al.: Near-infrared spectroscopy can predict the onset of cerebral hyperperfusion syndrome after carotid endarterectomy. Cerebrovasc Dis. 2012, 34:314-21. 10.1159/000343229

7. Pohl A, Cullen DJ: Cerebral ischemia during shoulder surgery in the upright position: a case series . J Clin Anesth. 2005, 17:463-9. 10.1016/j.jclinane.2004.09.012

8. Aguirre JA, Etzensperger F, Brada M, et al.: The beach chair position for shoulder surgery in intravenous general anesthesia and controlled hypotension: Impact on cerebral oxygenation, cerebral blood flow and neurobehavioral outcome. J Clin Anesth. 2019, 53:40-8. 10.1016/j.jclinane.2018.09.035

9. Park EY, Koo BN, Min KT, Nam SH: The effect of pneumoperitoneum in the steep Trendelenburg position on cerebral oxygenation. Acta Anaesthesiol Scand. 2009, 53:895-9. 10.1111/j.1399-6576.2009.01991.x

10. Closhen D, Treiber AH, Berres M, Sebastiani A, Werner C, Engelhard K, Schramm P: Robotic assisted prostatic surgery in the Trendelenburg position does not impair cerebral oxygenation measured using two different monitors: A clinical observational study. Eur J Anaesthesiol. 2014, 31:104-9. 10.1097/EJA.0000000000000000

11. Doe A, Kumagai M, Tamura Y, Sakai A, Suzuki K: A comparative analysis of the effects of sevoflurane and propofol on cerebral oxygenation during steep Trendelenburg position and pneumoperitoneum for roboticassisted laparoscopic prostatectomy. J Anesth. 2016, 30:949-55. 10.1007/s00540-016-2241-y

12. Jeong H, Jeong S, Lim HJ, Lee J, Yoo KY: Cerebral oxygen saturation measured by near-infrared spectroscopy and jugular venous bulb oxygen saturation during arthroscopic shoulder surgery in beach chair position under sevoflurane-nitrous oxide or propofol-remifentanil anesthesia. Anesthesiology. 2012, 116:1047-56. 10.1097/ALN.0b013e31825154d2

13. Kim JY, Lee JS, Lee KC, Kim HS, Kim SH, Kwak HJ: The effect of desflurane versus propofol on regional cerebral oxygenation in the sitting position for shoulder arthroscopy. J Clin Monit Comput. 2014, 28:371-6. 10.1007/s10877-013-9543-5

14. Takechi K, Kitamura S, Shimizu I, Yorozuya T: Lower limb perfusion during robotic-assisted laparoscopic radical prostatectomy evaluated by near-infrared spectroscopy: an observational prospective study. BMC Anesthesiol. 2018, 18:114. 10.1186/s12871-018-0567-8

15. Myburgh JA, Upton RN, Grant C, Martinez A: The cerebrovascular effects of adrenaline, noradrenaline and dopamine infusions under propofol and isoflurane anaesthesia in sheep. Anaesth Intensive Care. 2002, 30:725-33. 10.1177/0310057X0203000602

16. Hayashi K, Tanabe K, Minami K, Sakata K, Nagase K, Iida H: Effect of blood pressure elevation on cerebral oxygen desaturation in the beach chair position. Asian J Anesthesiol. 2017, 55:13-6. 10.1016/j.aja.2017.05.007

17. Lassen NA: Cerebral blood flow and oxygen consumption in man. Physiol Rev. 1959, 39:183-238. 10.1152/physrev.1959.39.2.183

18. Strandgaard S: Autoregulation of cerebral blood flow in hypertensive patients. The modifying influence of prolonged antihypertensive treatment on the tolerance to acute, drug-induced hypotension. Circulation. 1976, 53:720-7. 10.1161/01.cir.53.4.720

19. Kalmar AF, Dewaele F, Foubert L, Hendrickx JF, Heeremans EH, Struys MM, Absalom A: Cerebral haemodynamic physiology during steep Trendelenburg position and $\mathrm{CO}(2)$ pneumoperitoneum. $\mathrm{Br} \mathrm{J}$ Anaesth. 2012, 108:478-84. 10.1093/bja/aer448

20. Falabella A, Moore-Jeffries E, Sullivan MJ, Nelson R, Lew M: Cardiac function during steep Trendelenburg position and $\mathrm{CO} 2$ pneumoperitoneum for robotic-assisted prostatectomy: a trans-oesophageal Doppler probe study. Int J Med Robot. 2007, 3:312-5. 10.1002/rcs.165

21. Soeding PF, Wang J, Hoy G, Jarman P, Phillips H, Marks P, Royse C: The effect of the sitting upright or 'beachchair' position on cerebral blood flow during anaesthesia for shoulder surgery. Anaesth Intensive Care. 2011, 39:440-8. 10.1177/0310057X1103900315

22. Weiskopf RB, Cahalan MK, Eger EI 2nd, et al.: Cardiovascular actions of desflurane in normocarbic volunteers. Anesth Analg. 1991, 73:143-56.

23. Conti A, Iacopino DG, Fodale V, Micalizzi S, Penna O, Santamaria LB: Cerebral haemodynamic changes during propofol-remifentanil or sevoflurane anaesthesia: transcranial Doppler study under bispectral index monitoring. Br J Anaesth. 2006, 97:333-9. 10.1093/bja/ael169

24. Bedforth NM, Girling KJ, Skinner HJ, Mahajan RP: Effects of desflurane on cerebral autoregulation . Br J Anaesth. 2001, 87:193-7. 10.1093/bja/87.2.193 